\title{
Computational Investigations of Solid-Liquid Interfaces
}

\section{Final Report}

DOE/Office of Science Program Office: Basic Energy Sciences

DOE/Office of Science Program Office Technical Contact: Dr. Jane Zhu, Dr. Bonnie Gersten

DOE Grant Number: DE-FG02-06ER46282

Project Period: $5 / 1 / 06-4 / 30 / 11$

Principal Investigator

Mark Asta

Department of Chemical Engineering and Materials Science, University of California, Davis Department of Materials Science and Engineering, University of California, Berkeley

Email:mdasta@berkeley.edu

Phone: (510) 643-9631

Recipient Organization

Office for Research

Sponsored Programs

University of California - Davis

One Shields Ave, Davis, CA 95616

\section{$\underline{\text { Table of Contents }}$}

1. Project Overview ......................................................................................................2

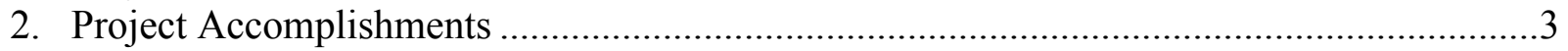

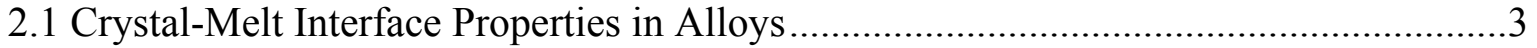

2.2 Faceted Solid-Liquid Interfaces .......................................................................4

2.3 Non-Equilibrium Kinetic Processes at Alloy Crystal-Melt Interfaces ..........................6

2.4 Grain Boundaries at High Homologous Temperatures...............................................6

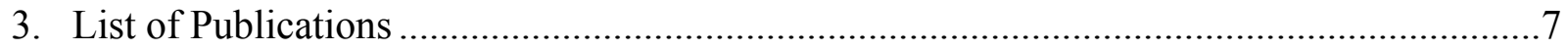




\section{Project Overview}

In a variety of materials synthesis and processing contexts, atomistic processes at heterophase interfaces play a critical role governing defect formation, growth morphologies, and microstructure evolution. Accurate knowledge of interfacial structure, free energies, mobilities and segregation coefficients are critical for predictive modeling of microstructure evolution, yet direct experimental measurement of these fundamental interfacial properties remains elusive in many cases. In this project first-principles calculations were combined with molecular-dynamics (MD) and Monte-Carlo (MC) simulations, to investigate the atomic-scale structural and dynamical properties of heterophase interfaces, and the relationship between these properties and the calculated thermodynamic and kinetic parameters that influence the evolution of phase transformation structures at nanometer to micron length scales. The topics investigated in this project were motivated primarily by phenomena associated with solidification processing of metals and alloys, and the main focus of the work was thus on solid-liquid interfaces and hightemperature grain boundaries. Additional efforts involved first-principles calculations of coherent solid-solid heterophase interfaces, where a close collaboration with researchers at the National Center for Electron Microscopy was undertaken to understand the evolution of novel core-shell precipitate microstructures in aluminum alloys. The next section reviews the main accomplishments under this project.

\section{Project Accomplishments}

2.1 Crystal-Melt Interface Properties in Alloys: In the development of the modern theoretical framework for understanding dendrite growth, attention focused initially almost exclusively on the relationship between the steady-state tip radius and velocity of a growing dendrite. An issue of equal fundamental and practical importance, that received considerably less attention until recently, is the question of the crystalline orientation selected by the growing dendrite tip. This orientation selection affects the overall symmetry of the growing dendrite and is an important factor governing the morphology and growth rate of solidification microstructures. In most materials dendrite tips choose high-symmetry growth directions, leading to six-fold symmetry for dendrites growing in the basal planes of hexagonal snow crystals and many hep metals, and four-fold symmetry for most cubic metals growing along $<100>$ directions. However, a growing number of observations have been reported where alloying has been found to induce dramatic changes in solidification morphologies, leading to highly complex growth shapes that remain incompletely understood. In the most detailed study of this phenomenon to date, Haxhimali et al. ${ }^{1}$ showed that over a range of $\mathrm{Zn}$ compositions, the dendrite growth direction, in commerically relevant Al-Zn alloys, changes smoothly from $<100>$ to $<110>$. To interpret the experimental results the authors performed phase-field simulations of dendrite growth where both the four-fold and six-fold parameters characterizing the anisotropy in the crystal-melt interfacial free energy $(\gamma)$ were varied. The modeling study showed that in the space of these two anisotropy parameters there exist regions where $\langle 100\rangle$ and $\langle 110\rangle$ growth are preferred, as well as an intermediate "hyperbranched" region where the misorientation angle, as measured from the $<100>$ growth direction, varies smoothly from 0 to $\pi / 4$. A key assumption in this theoretical interpretation is

\footnotetext{
${ }^{1}$ T. Haxhimali, A. Karma, F. Gonzales and M. Rappaz, "Orientation selection in dendritic evolution," Nature Mat. 5, 660-664 (2006).
} 
that alloying can lead to changes in the anisotropies of $\gamma$ strong enough to induce changes in dendrite growth direction. Prior to the efforts under this project there was no direct evidence either from experiment or simulation that this was the case. Work completed in this project $[2,13]$ yielded new insights into the nature of alloying effects on the free energies of crystal-melt interfaces, and demonstrated that indeed composition-induced variations in $\gamma$ anisotropies can be significant on the scale required to induce changes in dendrite growth directions, even in an alloy system with fairly ideal solution-thermodynamic properties.

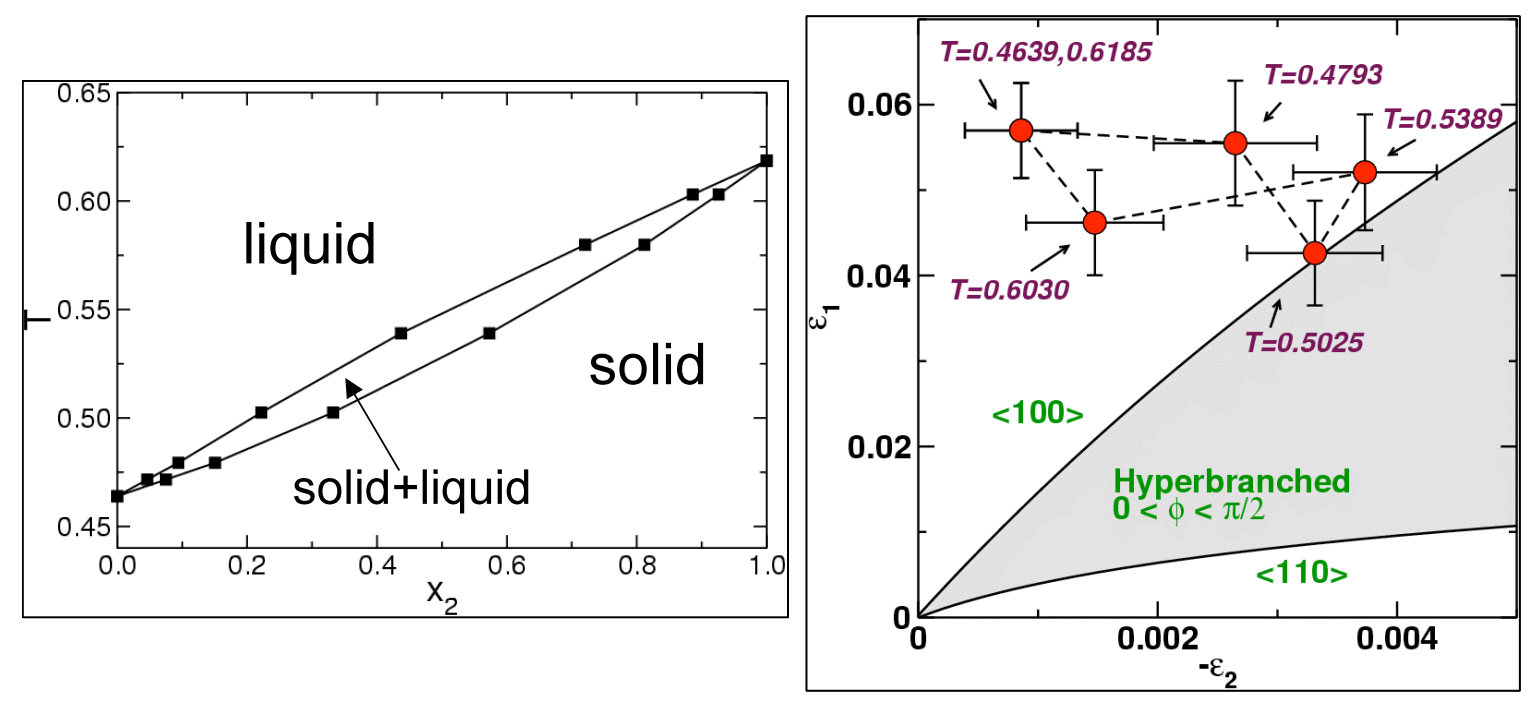

(a)

(b)

Figure 1: Results of atomistic calculations of equilibrium solid-liquid phase boundaries (a) and crystal-melt interfacial free energy anisotropy parameters (b). Results are from work recently submitted to Physical Review Letters [6], and are described in further detail in the text.

The results of the modeling work [2,13] are summarized in Figure 1. In this work, which represents the first detailed calculation of the composition dependence of crystal-melt interfacial free energies in an alloy, we considered a model Lennard-Jones alloy system featuring zero sizemismatch and fairly ideal solution thermodynamics. This system provided an important benchmark, the results for which isolate the effects on crystal-melt interface properties arising from solute partitioning and the associated composition and density profiles across the solidliquid interface, without introducing complicating factors associated with size mismatch. The phase diagram for the system considered in this work featured a "lens" type solid-liquid phase diagram, shown in Fig. 1 (a), where the temperature is expressed in reduced Lennard-Jones units. The capillary-fluctuation method was employed to compute excess free energies between coexisting crystal and melt phases, with composition given by the corresponding solidus/liquidus boundaries. In Figure 1 (b) results of the calculations are plotted for the four-fold and six-fold anisotropy parameters, $\varepsilon_{1}$ and $\varepsilon_{2}$, respectively. These parameters, which characterize the dependence of $\gamma$ on interface normal, determine dendrite growth orientations in the limit of low undercoolings, as shown by the solid-lines representing results of the previous phase-field modeling by Haxhimali et al.. For large positive values of $\varepsilon_{1}$ and relatively small values of $\varepsilon_{2}$, dendrite growth along $<100>$ is preferred, as observed in most cubic materials. However, for small values of $\varepsilon_{1}$ and relatively large negative values of $\varepsilon_{2}$, dendrite growth along $<110>$ is 
favored, as observed in some Al alloy systems. The central question addressed in the atomistic work was whether the composition induced variations in these anisotropy parameters can be strong enough to lead to changes in growth orientation.

The results for Lennard-Jones alloys, plotted with red symbols in Fig. 1(b) suggested that this is indeed the case. While the pure element anisotropy parameters (indicated by $\mathrm{T}=0.4639$ and 0.6185) fall firmly inside the $<100>$ domain, the effect of alloying is seen to amplify the magnitude of the six-fold anisotropy parameter, increasing tendencies toward $<110>$ growth. For elemental metals such as Al, where the anisotropies have been predicted to lie close to the boundary between $\langle 100\rangle$ and hyperbranched growth [13], these variations in anisotropy parameters arising from compositional changes are seen to be strong enough to induce changes in growth orientation.

2.2 Faceted Solid-Liquid Interfaces: In solution growth, and growth of ionic or covalent solids from their melts or liquid catalysts, faceted interface morphologies are commonly observed. In these contexts crystal growth rates and morphologies are sensitively affected by the thermodynamic and kinetic properties of steps present on vicinal interfaces. In this project we applied MD simulations in detailed studies of crystal-melt interface steps, employing the Stillinger-Weber (SW) model of Si as a model system $[6,9,16]$.

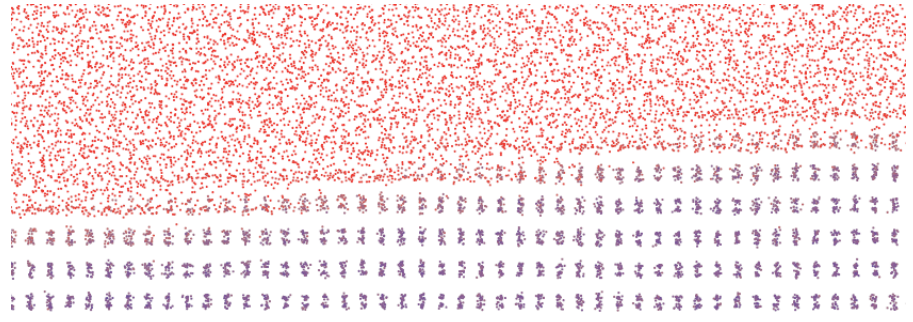

(a)

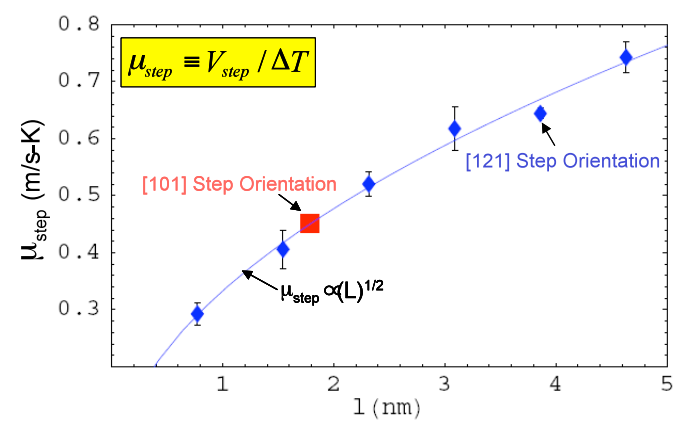

(b)

Figure 2: Snapshot of a simulation cell (a) showing steps at a faceted crystal-melt interface in Si. Results of nonequilibrium MD simulations are plotted in (b) showing calculated step mobilities as a function of step spacing (1).

Non-equilibrium molecular-dynamics (NEMD) methods were applied to investigate crystal growth at interfaces with orientations vicinal to the faceted [111] plane. Steps at this interface were found to have a double-height structure and are characterized as being highly diffuse structurally. In the NEMD simulations, a step-flow growth mode was verified and found to be stable up to undercoolings on the order of $30 \mathrm{~K}$. Step mobilities were calculated from simulations featuring a wide range of step spacings, from roughly 0.8 to $4.6 \mathrm{~nm}$. In this nanometer-scale range the step mobilities were found to display a pronounced dependence on step spacing, as show in Figure 2. It is observed that relatively strong step-step interactions give rise to a decrease in step roughness with decreasing step spacing, correlating with an associated decrease in step mobility. The magnitudes of the calculated step mobilities are found to be roughly an order of magnitude larger than the predictions of classical theories which assume that step motion is governed by the attachment of "adatoms" from the liquid to the solid at welldefined kink sites. In contrast to the classical picture, the simulations for SW-Si crystal-melt interfaces suggest that steps advance by rearrangements of the atoms on the layer above the crystal terrace that are already highly ordered relative to the liquid phase. Atoms in this 
interfacial layer are expected to be more easily able to rearrange themselves to form crystalline order (and thus advance the step forward), thus requiring a lower driving force than that assumed in the classical model to advance the step forward.

The tools developed in the work on SW-Si models were extended in simulation studies of size effects on nucleation kinetics in model systems crystallizing in confined geometries [16]. The work was motivated in the context of catalyst-mediated nanowire growth, where kinetic processes at the growth interface can play an important role in governing wire compositions, morphologies, and growth rates. Simulated growth rates displayed a dependence on nanowire diameter consistent with a size dependent barrier for facet nucleation. A theory for the interface mobility was developed, establishing a source for size-dependent growth rates that is an intrinsic feature of systems possessing growth interfaces with faceted and rough orientations.
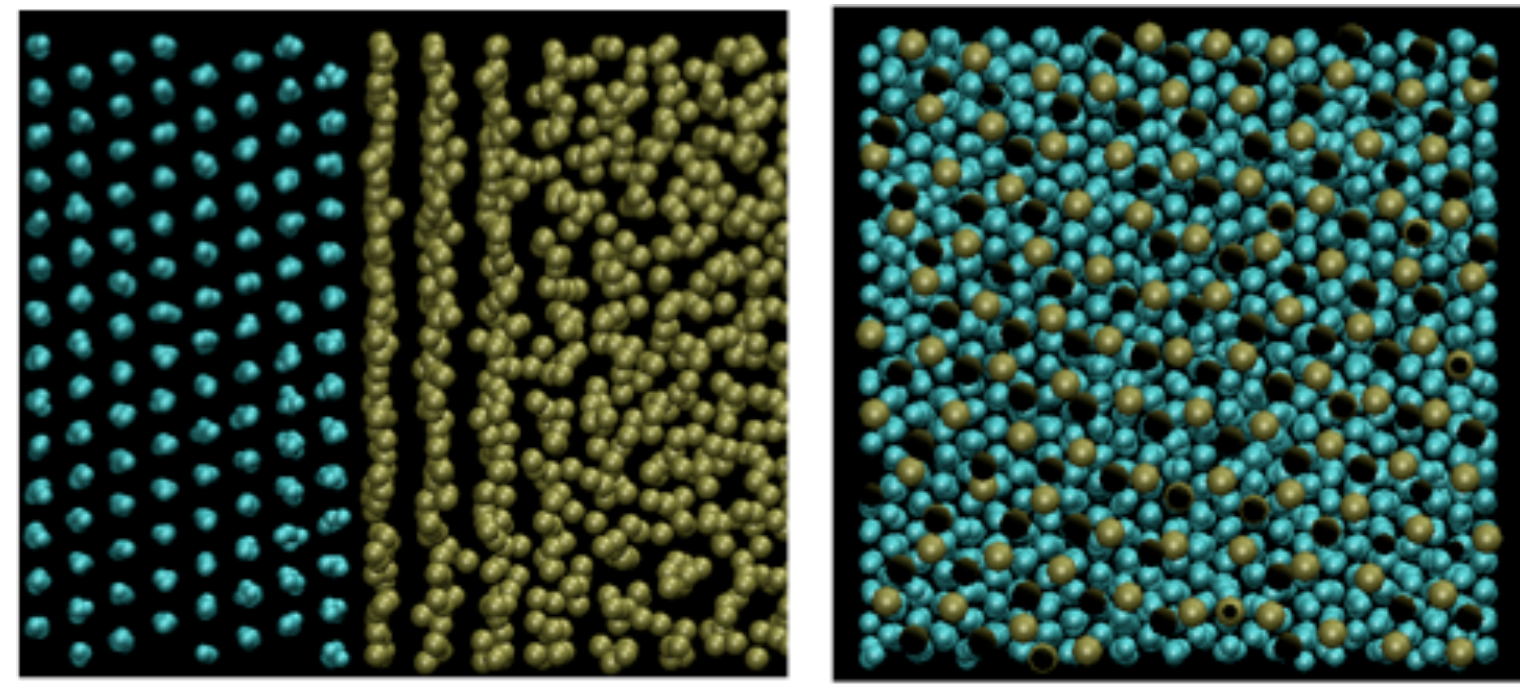

Figure 3: $\mathrm{MD}$ snapshots illustrating the interface structure for liquid $\mathrm{Pb}$ (gold spheres) against solid $\mathrm{Cu}$ (blue). The panel on the left shows a side view highlighting the layers of the liquid $\mathrm{Pb}$ against solid $\mathrm{Cu}$. The right panel shows the lateral order within the first two $\mathrm{Pb}$ interfacial layers: gold circles are the first-layer atoms and the black holes are the positions of the second layer atoms; blue denotes the $\mathrm{Cu}$ interfacial layer. The first-layer $\mathrm{Pb}$ atoms are seen to display a high degree of order, with a well-defined orientation relationship relative to the $\mathrm{Cu}$ (111) planes.

Our most recent work related to the properties of faceted crystal-melt interfaces has involved molecular-dynamics studies of chemically heterogeneous interfaces. The systems investigated in this work are $\mathrm{Pb} / \mathrm{Cu}$ [25] and $\mathrm{Pb} / \mathrm{Al}$. The work on these materials was motivated by experimental studies in the group of Uli Dahmen at the National Center for Electron Microscopy, in which in-situ electron microscopy studies of the Brownian motion of small embedded $\mathrm{Pb}$ liquid particles yield unique insights into the thermodynamic properties and kinetic processes governing the motion of faceted solid-liquid interfaces. Our work led to the identification of a novel pre-freezing layer in (111) oriented $\mathrm{Pb} / \mathrm{Cu}$ interfaces [25], Figure 3, that is absent for other crystalline orientations. Compared to $\mathrm{Pb} / \mathrm{Cu}$, our results for $\mathrm{Pb} / \mathrm{Al}$ show less ordering of the liquid at the interface, but a more pronounced degree of alloying in the interfacial layers. The $\mathrm{Al} / \mathrm{Pb}$ results have also been generalized in the first study of the structure and dynamics of steps at faceted, chemically-heterogeneous solid-liquid interfaces. A manuscript related to this work is currently under preparation. 
2.3 Non-Equilibrium Kinetic Processes at Alloy Crystal-Melt Interfaces: Under the nonequilibrium conditions characteristic of rapid solidification, solute effects on solidification microstructures arise due to their influence on interface mobilities, and the closely-related phenomenon of solute trapping. In this project we developed a non-equilibrium MD methodology for the study of such properties. A major technical issue that has been addressed in this work is related to the large thermal gradients induced in rapid growth simulations, due to the release of latent heat $[18,22,23]$. The methods that have been developed in this work were applied to a model Lennard-Jones alloy system featuring large solute partitioning between solid and liquid phases, and the Ni-Cu alloy system modeled by embedded-atom-method potentials [26]. The relationships between the interface velocity, thermodynamic driving force, and solute partition coefficient were computed and analyzed within the framework of kinetic theories accounting for solute trapping and solute drag. The results show a transition to complete solute trapping at high growth velocities, establish appreciable solute drag at low growth velocities, and provide insights into the nature of crystalline anisotropies and solute effects on interface mobilities. Specifically, we demonstrated anisotropy in the crystal-melt interface mobility comparable to that obtained for elemental fcc-forming systems, while showing a tendency for reduced values of the mobility in alloys relative to corresponding pure element systems. The MD data also indicated the presence of appreciable solute drag for the systems considered.

2.4 Grain Boundaries at High Homologous Temperatures: Surface premelting - the formation of thin, liquid-like films on solid surfaces at temperatures below the melting point - is a well established phenomenon with important consequences for many natural processes. Premelting at grain boundaries - the formation of nanometer-scale films with liquid-like properties confined between misoriented grains - can have similarly important consequences in the context of materials processing and service, by dramatically altering the properties of polycrystals at high temperatures. To understand outstanding interfacial phase-transition aspects underlying this phenomenon, we have undertaken collaborative research under the Computational Materials Science Network program that led to the developed of new computational methods for quantitative studies of grain boundary premelting. This effort led to fundamental new insights into the nature of the "disjoining forces" that energetically favor the formation of premelted films by effectively pushing apart crystal-liquid interfaces. [12,20,21,24,28].

Molecular dynamics simulations were applied in a comparative study of (i) a $\Sigma 9\langle 115\rangle 120^{\circ}$ twist and (ii) a $\Sigma 9\langle 110\rangle\{411\}$ symmetric tilt boundary in a classical embedded-atom model of elemental Ni. Although both boundaries feature highly disordered structures near the melting point, the nature of the temperature dependence of the width of the disordered regions in these boundaries is qualitatively different, as illustrated in Figure 4 (a). The former boundary displays behavior consistent with a logarithmically diverging premelted layer thickness as the melting temperature is approached from below, while the latter displays behavior featuring a finite grainboundary width at the melting point. It was demonstrated that both types of behavior can be quantitatively described within a sharp-interface thermodynamic formalism involving a widthdependent interfacial free energy, referred to as the disjoining potential. The disjoining potential for boundary (i) is calculated to display a monotonic exponential dependence on width, while that of boundary (ii) features a weak attractive minimum. Boundary (ii) is found to feature a structure at high homologous temperatures characterized by localized regions of disorder, separated by highly ordered "solid bridges," as illustrated in Figure 4 (b). 


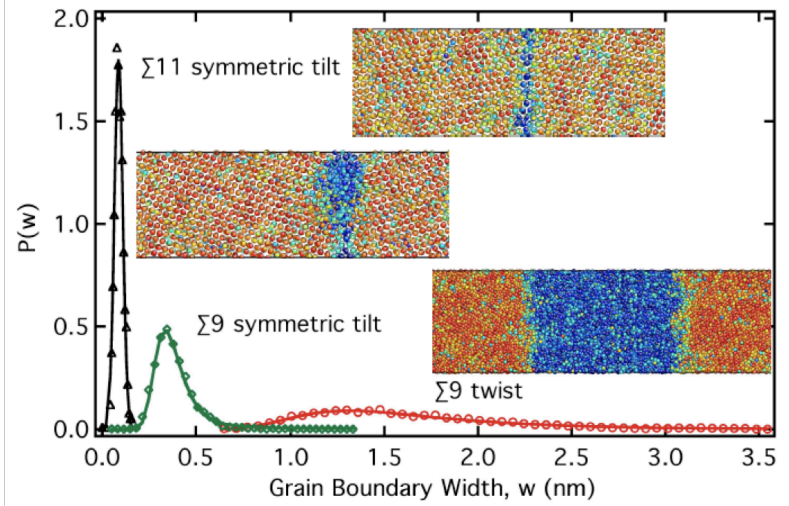

(a)

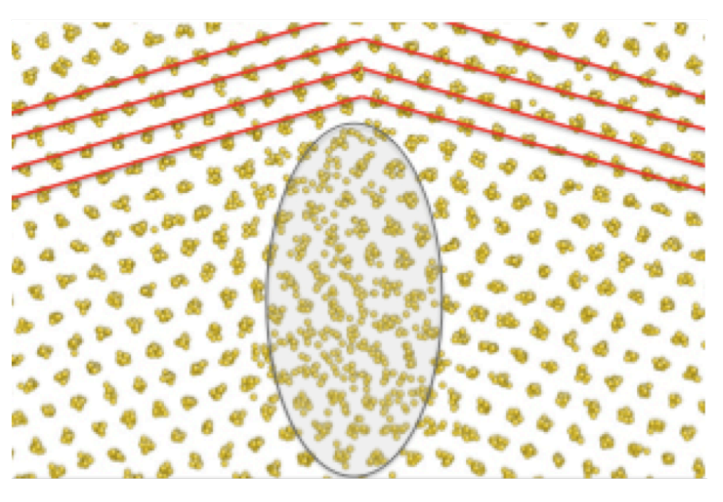

(b)

Figure 4: The figure on the left (a) displays MD snapshots and equilibrium grain-boundary width histograms for three boundaries displaying qualitatively distinct structures at high homologous temperatures. The $\Sigma 11$ tilt boundary remains highly ordered while the $\Sigma 9$ twist boundary displays a large premelted layer. The $\Sigma 9$ tilt boundary shows intermediate behavior, illustrated in (b), characterized by the formation of localized regions of disorder separated by ordered "solid bridges".

In similar studies for bcc metals, we reported the finding of a novel grain-boundary structural phase transition in both molecular-dynamics and phase-field-crystal simulations of classical models of $\mathrm{Fe}$ [24]. This transition is characterized by pairing of individual dislocations with mixed screw and edge components. It was found that this type of transition is driven by a combination of factors including elastic softening, core interaction, and core disordering. At high homologous temperatures the occurrence of this transition is shown to prevent premelting at misorientation angles where it would otherwise be expected.

\section{List of Publications}

[1] Z. G. Xia, D. Y. Sun, M. Asta and J. J. Hoyt, "Molecular Dynamics Calculations of the Crystal-Melt Interfacial Mobility for Hexagonal-Close-Packed Mg," Phys. Rev. B 75, 012103 (2007).

[2] C. A. Becker, D. Olmsted, M. Asta, J. J. Hoyt, and S. M. Foiles, "Atomistic Underpinnings for Orientation Selection in Alloy Dendritic Growth," Phys. Rev. Lett. 98, 125701 (2007).

[3] J. Z. Liu, G. Ghosh, A. van de Walle and M. Asta, "Transferable Force-Constant Modeling of Vibrational Thermodynamic Properties in FCC-Based Al-TM (TM=Ti, Zr, Hf) Alloys," Phys. Rev. B 75, 104117 (2007). [Note: paper acknowledged the old program number or this project: DE-FG02-01ER45910.]

[4] G. Ghosh, A. van de Walle and M. Asta, "First-Principles Phase Stability Calculations of Pseudobinary Alloys of (Al,Zn)3Ti with L12, D022 and D023 Structures," J. Phase Equilibria and Diffusion 28, 9-22 (2007). [Note: paper acknowledged the old program number or this project: DE-FG02-01ER45910.] 
[5] C. A. Becker, D. Buta, J. J. Hoyt and M. Asta, "Crystal-Melt Interface Stresses: Atomistic Simulation Calculations for a Lennard-Jones Alloy, Stillinger-Weber Si, and Embedded Atom Method Ni," Phys. Rev. E 75, 061610 (2007).

[6] D. Buta, M. Asta and J. J. Hoyt, "Kinetic Coefficient of Steps at the Si(111) Crystal-Melt Interface from Molecular Dynamics Simulations," J. Chem. Phys. 127, 074703 (2007).

[7] G. Ghosh, A. van de Walle, and M. Asta, "First-Principles Calculations of Structural and Energetic Properties of BCC, FCC and HCP Solid Solutions in the Al-TM (TM=Ti,Zr,Hf) Systems: A Comparison of Cluster Expansion and Supercell Methods," Acta Mater. 56, 3202-3221 (2008).

[8] M. I. Mendelev, M. J. Kramer, C. A. Becker and M. Asta, "Analysis of Semi-Empirical Interatomic Potentials Appropriate for Simulation of Crystalline and Liquid $\mathrm{Al}$ and $\mathrm{Cu}$," Phil. Mag. 88, 1723-1750 (2008).

[9] D. Buta, M. Asta and J. J. Hoyt, "Structure and Dynamics of a Faceted Crystal-Melt Interface," Phys. Rev. E 78, 031605 (2008).

[10] S. Angioletti-Uberti, M. Asta, M. W. Finnis and P. D. Lee, "Solid-Liquid Phase Equilibria from Free Energy Perturbation Calculations," Phys. Rev. B 78, 134203 (2008).

[11] M. Asta, C. Beckermann, A. Karma, W. Kurz, R. Napolitano, M. Plapp, G. Purdy, M. Rappaz, and R. Trivedi, "Solidification Microstructures and Solid State Parallels: Recent Developments, Future Directions," Acta Mater. 57, 941-971 (2009).

[12] J. J. Hoyt, D. Olmsted, S. Jindal, M. Asta, and A. Karma, "Method for Computing ShortRange Forces Driving Grain Boundary Premelting," Phys. Rev. E 79, 020601(R) (2009). [Note: paper acknowledged the old program number or this project: DE-FG0201ER45910.]

[13] C. A. Becker, D. L. Olmsted, M. Asta, J. J. Hoyt and S. M. Foiles, "Atomistic Simulations of Crystal-Melt Interfaces in a Model Binary Alloy: Interfacial Free Energies, Adsorption Coefficients and Excess Entropy,” Phys. Rev. B 79, 054109 (2009).

[14] M. D. Rossell, R. Erni, M. Asta, V. Radmilovic and U. Dahmen, "Atomic-Resolution Imaging of Lithium in $\mathrm{Al}_{3} \mathrm{Li}$ Precipitates," Phys. Rev. B 80, 024110 (2009).

[15] B. B. Laird, R. L. Davidchack, Y. Yang and M. Asta, "Determination of the Solid-Liquid Interfacial Free Energy Along a Coexistence Line by Gibbs-Cahn Integration," J. Chem. Phys. 131, 114110 (2009).

[16] T. Haxhimali, D. Buta, M. Asta, P. W. Voorhees and J. J. Hoyt, "Size-Dependent Nucleation Kinetics at Non-planar Nanowire Growth Interfaces," Phys. Rev. E 80, 050601(R) (2009). 
[17] M. I. Mendelev, M. Asta, M. J. Rahman and J. J. Hoyt, "Development of Interatomic Potentials Appropriate for Simulation of Solid-Liquid Interface Properties in Al-Mg Alloys," Phil. Mag. 89, 3269-3285 (2009).

[18] J. Monk, Y. Yang, M. I. Mendelev, M. Asta, J. J. Hoyt, and D. Y. Sun, "Determination of the Crystal-Melt Interface Kinetic Coefficient from Molecular-Dynamics Simulations," Modelling Simul. Mater. Sci. Eng. 18, 015004 (2010).

[19] Y. Mishin, M. Asta and J. Li, "Atomistic Modeling of Interfaces and Their Impact on Microstructure and Properties," Acta Mater. 58, 1117-1151 (2010).

[20] S. J. Fensin, D. Olmsted, D. Buta, M. Asta, A. Karma and J. J. Hoyt, "Structural Disjoining Potential for Grain Boundary Premelting and Grain Coalescence from Molecular-Dynamics Simulations," Phys. Rev. E 81, 031601 (2010).

[21] H. Song, S. J. Fensin, M. Asta, and J. J. Hoyt, “A Molecular Dynamics Simulation of (110) Surface Premelting in Ni," Scripta Mater. 63, 128-131 (2010).

[22] M. I. Mendelev, M. J. Rahman, J. J. Hoyt and M. Asta, "Molecular Dynamics Study of Solid-Liquid Interface Migration in fcc Metals," Modelling Simul. Mater. Sci. Eng. 18, 074002 (2010).

[23] Y. F. Gao, Y. Yang, D. Y. Sun, M. Asta and J. J. Hoyt, "Molecular Dynamics Simulations of the Crystal-Melt Interface Mobility in HCP Mg and BCC Fe," J. Crystal Growth 312, 3238-3242 (2010).

[24] D. L. Olmsted, D. Buta, A. Adland, S. M. Foiles, M. Asta, and A. Karma, "DislocationPairing Transitions in Hot Grain Boundaries," Phys. Rev. Lett. 106, 046101 (2011).

[25] J. P. Palafox-Hernandez, B. B. Laird and M. Asta, "Atomistic Characterization of the CuPb Solid-Liquid Interface,” Acta Mater. 59, 3137-3144 (2011).

[26] Y. Yang, H. Humadi, D. Buta, B.B. Laird, D.Y. Sun, J.J. Hoyt and M. Asta, "Atomistic Simulations of Non-Equilibrium Crystal-Growth Kinetics from Alloy Melts," Phys. Rev. Lett. 107, 025505 (2011).

[27] V. Radmilovic, C. Ophus, E.A. Marquis, M.D. Rossell, A. Tolley, A. Gautam, M. Asta and U. Dahmen, "Highly Monodisperse Core-Shell Particles Created by Solid-State Reactions," Nature Materials, doi 10.1038/nmat3077.

[28] Saryu Jindal Fensin, "Molecular Dynamics Study of Grain Boundary Structure and Properties at High Temperatures," PhD Thesis, Department of Chemical Engineering and Materials Science, University of California, Davis (2010). 